\title{
Average Values of Triangles
}

\author{
Péter Kórus ${ }^{1}$
}

${ }^{1}$ Department of Mathematics, Juhász Gyula Faculty of Education, University of Szeged, Hattyas utca 10, H-6725 Szeged, Hungary

\section{Article Info}

Keywords: Area, Circumradius, Inradius, Perimeter, Triangle 2010 AMS: 51 M04

Received: 22 February 2020

Accepted: 20 April 2020

Available online: 10 June 2020

\begin{abstract}
The average area and perimeter of triangles defined by three random points on the circumference of a unit circle are already known. In this paper, we calculate the average value of the inradius along with its variance. We also consider the average area, inradius and circumradius of triangles of unit perimeter.
\end{abstract}

\section{Triangles of unit circumradius}

How to choose a triangle randomly on the circumference of a unit circle? An answer is selecting three points $A, B, C$ on the circumference uniformly. Many questions arise regarding these triangles $A B C$. What is the average area or average perimeter or average inradius of the triangles? The average area of these triangles is known to be $\frac{3}{2 \pi}=0.47746 \ldots$, see [2], while the average perimeter is $\frac{12}{\pi}=3.81971 \ldots$, see [1]. Our goal is to calculate the average value of the inradius of the triangles. We use the same characterization of the triangle as in [2]. Suppose without loss of generality that the center of the circumcircle of the triangle is $O(0,0)$, one vertex is $A(1,0)$ and the other two vertices are determined by directed angles $\angle A O B=\theta_{1} \in[0, \pi]$ and $\angle A O C=\theta_{2} \in[0,2 \pi)$. Then the inradius of the triangle is equal to

$$
r\left(\theta_{1}, \theta_{2}\right)=r(\triangle A B C)=\frac{A(\triangle A B C)}{\frac{P(\triangle A B C)}{2}}=\frac{2 \sin \frac{\theta_{1}}{2} \sin \frac{\theta_{2}}{2}\left|\sin \frac{\theta_{2}-\theta_{1}}{2}\right|}{\sin \frac{\theta_{1}}{2}+\sin \frac{\theta_{2}}{2}+\left|\sin \frac{\theta_{2}-\theta_{1}}{2}\right|} .
$$

We calculate the average value of the inradius. First note that

$$
\begin{aligned}
\bar{r} & =\frac{1}{\pi^{2}} \int_{0}^{\pi} \int_{0}^{2 \pi} \frac{\sin \frac{\theta_{1}}{2} \sin \frac{\theta_{2}}{2}\left|\sin \frac{\theta_{2}-\theta_{1}}{2}\right|}{\sin \frac{\theta_{1}}{2}+\sin \frac{\theta_{2}}{2}+\left|\sin \frac{\theta_{2}-\theta_{1}}{2}\right|} d \theta_{2} d \theta_{1} \\
& =\frac{1}{\pi^{2}} \int_{0}^{\pi} \int_{\theta_{1}}^{2 \pi} \frac{\sin \frac{\theta_{1}}{2} \sin \frac{\theta_{2}}{2} \sin \frac{\theta_{2}-\theta_{1}}{2}}{\sin \frac{\theta_{1}}{2}+\sin \frac{\theta_{2}}{2}+\sin \frac{\theta_{2}-\theta_{1}}{2}} d \theta_{2} d \theta_{1}+\frac{1}{\pi^{2}} \int_{0}^{\pi} \int_{0}^{\theta_{1}} \frac{\sin \frac{\theta_{1}}{2} \sin \frac{\theta_{2}}{2} \sin \frac{\theta_{1}-\theta_{2}}{2}}{\sin \frac{\theta_{1}}{2}+\sin \frac{\theta_{2}}{2}+\sin \frac{\theta_{1}-\theta_{2}}{2}} d \theta_{2} d \theta_{1} \\
& =\frac{1}{\pi^{2}}\left[\int_{0}^{\pi} I_{1} d \theta_{1}+\int_{0}^{\pi} I_{2} d \theta_{1}\right] .
\end{aligned}
$$


Simplify the integrand in $I_{1}$ as follows.

$$
\begin{aligned}
\frac{\sin \frac{\theta_{1}}{2} \sin \frac{\theta_{2}}{2} \sin \frac{\theta_{2}-\theta_{1}}{2}}{\sin \frac{\theta_{1}}{2}+\sin \frac{\theta_{2}}{2}+\sin \frac{\theta_{2}-\theta_{1}}{2}} & =\frac{2 \sin \frac{\theta_{1}}{2} \sin \frac{\theta_{2}}{2} \sin \frac{\theta_{2}-\theta_{1}}{4} \cos \frac{\theta_{2}-\theta_{1}}{4}}{2 \sin \frac{\theta_{2}+\theta_{1}}{4} \cos \frac{\theta_{2}-\theta_{1}}{4}+2 \sin \frac{\theta_{2}-\theta_{1}}{4} \cos \frac{\theta_{2}-\theta_{1}}{4}} \\
& =\frac{\sin \frac{\theta_{1}}{2} \sin \frac{\theta_{2}}{2} \sin \frac{\theta_{2}-\theta_{1}}{4}}{\sin \frac{\theta_{2}+\theta_{1}}{4}+\sin \frac{\theta_{2}-\theta_{1}}{4}} \\
& =\frac{4 \sin \frac{\theta_{1}}{4} \cos \frac{\theta_{1}}{4} \sin \frac{\theta_{2}}{4} \cos \frac{\theta_{2}}{4} \sin \frac{\theta_{2}-\theta_{1}}{4}}{2 \sin \frac{\theta_{2}}{4} \cos \frac{\theta_{1}}{4}} \\
& =2 \sin \frac{\theta_{1}}{4} \cos \frac{\theta_{2}}{4} \sin \frac{\theta_{2}-\theta_{1}}{4}
\end{aligned}
$$

Analogously, for the integrand in $I_{2}$,

$$
\frac{\sin \frac{\theta_{1}}{2} \sin \frac{\theta_{2}}{2} \sin \frac{\theta_{1}-\theta_{2}}{2}}{\sin \frac{\theta_{1}}{2}+\sin \frac{\theta_{2}}{2}+\sin \frac{\theta_{1}-\theta_{2}}{2}}=2 \cos \frac{\theta_{1}}{4} \sin \frac{\theta_{2}}{4} \sin \frac{\theta_{1}-\theta_{2}}{4} .
$$

Using (1.1), we calculate $I_{1}$ similarly as in [2]:

$$
\begin{aligned}
I_{1} & =2 \sin \frac{\theta_{1}}{4} \int_{\theta_{1}}^{2 \pi} \cos \frac{\theta_{2}}{4} \sin \frac{\theta_{2}-\theta_{1}}{4} d \theta_{2} \\
& =2 \sin \frac{\theta_{1}}{4} \int_{\theta_{1}}^{2 \pi} \cos \frac{\theta_{2}}{4}\left[\sin \frac{\theta_{2}}{4} \cos \frac{\theta_{1}}{4}-\sin \frac{\theta_{1}}{4} \cos \frac{\theta_{2}}{4}\right] d \theta_{2} \\
& =2 \sin \frac{\theta_{1}}{4} \cos \frac{\theta_{1}}{4} \int_{\theta_{1}}^{2 \pi} \cos \frac{\theta_{2}}{4} \sin \frac{\theta_{2}}{4} d \theta_{2}-2 \sin ^{2} \frac{\theta_{1}}{4} \int_{\theta_{1}}^{2 \pi} \cos ^{2} \frac{\theta_{2}}{4} d \theta_{2} \\
& =\sin \frac{\theta_{1}}{2}\left[-\cos \frac{\theta_{2}}{2}\right]_{\theta_{1}}^{2 \pi}-2 \sin ^{2} \frac{\theta_{1}}{4}\left[\sin \frac{\theta_{2}}{2}+\frac{\theta_{2}}{2}\right]_{\theta_{1}}^{2 \pi} \\
& =\sin \frac{\theta_{1}}{2}\left[1+\cos \frac{\theta_{1}}{2}+2 \sin ^{2} \frac{\theta_{1}}{4}\right]-2 \pi \sin ^{2} \frac{\theta_{1}}{4}+\theta_{1} \sin ^{2} \frac{\theta_{1}}{4} \\
& =2 \sin \frac{\theta_{1}}{2}-2 \pi \sin ^{2} \frac{\theta_{1}}{4}+\theta_{1} \sin ^{2} \frac{\theta_{1}}{4},
\end{aligned}
$$

while

$$
I_{2}=2 \cos \frac{\theta_{1}}{4} \int_{0}^{\theta_{1}} \sin \frac{\theta_{2}}{4} \sin \frac{\theta_{1}-\theta_{2}}{4} d \theta_{2}=2 \sin \frac{\theta_{1}}{2}-\theta_{1} \cos ^{2} \frac{\theta_{1}}{4} .
$$

Therefore, integration by parts gives us

$$
\begin{aligned}
\int_{0}^{\pi} I_{1} d \theta_{1} & =\int_{0}^{\pi} 2 \sin \frac{\theta_{1}}{2}-\pi\left(1-\cos \frac{\theta_{1}}{2}\right)+\frac{\theta_{1}}{2}\left(1-\cos \frac{\theta_{1}}{2}\right) d \theta_{1} \\
& =\left[-4 \cos \frac{\theta_{1}}{2}-\pi \theta_{1}+2 \pi \sin \frac{\theta_{1}}{2}+\frac{\theta_{1}^{2}}{4}\right]_{0}^{\pi}-\left[\theta_{1} \sin \frac{\theta_{1}}{2}\right]_{0}^{\pi}+\int_{0}^{\pi} \sin \frac{\theta_{1}}{2} d \theta_{1} \\
& =-\pi^{2}+2 \pi+\frac{\pi^{2}}{4}+4-\pi+2=6+\pi-\frac{3}{4} \pi^{2}
\end{aligned}
$$

and analogously,

$$
\begin{aligned}
\int_{0}^{\pi} I_{2} d \theta_{1} & =\int_{0}^{\pi} 2 \sin \frac{\theta_{1}}{2}-\frac{\theta_{1}}{2}\left(1+\cos \frac{\theta_{1}}{2}\right) d \theta_{1} \\
& =\left[-4 \cos \frac{\theta_{1}}{2}-\frac{\theta_{1}^{2}}{4}\right]_{0}^{\pi}-\left[\theta_{1} \sin \frac{\theta_{1}}{2}\right]_{0}^{\pi}+\int_{0}^{\pi} \sin \frac{\theta_{1}}{2} d \theta_{1} \\
& =6-\pi-\frac{1}{4} \pi^{2},
\end{aligned}
$$

and we obtain the average value of the inradius as follows.

\section{Formula 1.}

$$
\bar{r}=\frac{1}{\pi^{2}}\left[\int_{0}^{\pi} I_{1} d \theta_{1}+\int_{0}^{\pi} I_{2} d \theta_{1}\right]=\frac{12}{\pi^{2}}-1=0.21585 \ldots
$$

\subsection{Variance of the inradius}

Calculate the second moment of the inradius.

$$
\begin{aligned}
\overline{r^{2}} & =\frac{2}{\pi^{2}} \int_{0}^{\pi} \int_{\theta_{1}}^{2 \pi} \frac{\sin ^{2} \frac{\theta_{1}}{2} \sin ^{2} \frac{\theta_{2}}{2} \sin ^{2} \frac{\theta_{2}-\theta_{1}}{2}}{\left(\sin \frac{\theta_{1}}{2}+\sin \frac{\theta_{2}}{2}+\sin \frac{\theta_{2}-\theta_{1}}{2}\right)^{2}} d \theta_{2} d \theta_{1}+\frac{2}{\pi^{2}} \int_{0}^{\pi} \int_{0}^{\theta_{1}} \frac{\sin ^{2} \frac{\theta_{1}}{2} \sin ^{2} \frac{\theta_{2}}{2} \sin ^{2} \frac{\theta_{1}-\theta_{2}}{2}}{\left(\sin \frac{\theta_{1}}{2}+\sin \frac{\theta_{2}}{2}+\sin \frac{\theta_{1}-\theta_{2}}{2}\right)^{2}} d \theta_{2} d \theta_{1} \\
& =\frac{2}{\pi^{2}}\left[\int_{0}^{\pi} J_{1} d \theta_{1}+\int_{0}^{\pi} J_{2} d \theta_{1}\right] .
\end{aligned}
$$


Simplification for the integrand in $J_{1}$ is

and for the integrand in $J_{2}$ is

$$
\frac{\sin ^{2} \frac{\theta_{1}}{2} \sin ^{2} \frac{\theta_{2}}{2} \sin ^{2} \frac{\theta_{2}-\theta_{1}}{2}}{\left(\sin \frac{\theta_{1}}{2}+\sin \frac{\theta_{2}}{2}+\sin \frac{\theta_{2}-\theta_{1}}{2}\right)^{2}}=4 \sin ^{2} \frac{\theta_{1}}{4} \cos ^{2} \frac{\theta_{2}}{4} \sin ^{2} \frac{\theta_{2}-\theta_{1}}{4}
$$

$$
\frac{\sin ^{2} \frac{\theta_{1}}{2} \sin ^{2} \frac{\theta_{2}}{2} \sin ^{2} \frac{\theta_{1}-\theta_{2}}{2}}{\left(\sin \frac{\theta_{1}}{2}+\sin \frac{\theta_{2}}{2}+\sin \frac{\theta_{1}-\theta_{2}}{2}\right)^{2}}=4 \cos ^{2} \frac{\theta_{1}}{4} \sin ^{2} \frac{\theta_{2}}{4} \sin ^{2} \frac{\theta_{1}-\theta_{2}}{4}
$$

Calculate $J_{1}$ as follows:

$$
\begin{aligned}
J_{1} & =\frac{1}{2}\left(1-\cos \frac{\theta_{1}}{2}\right) \int_{\theta_{1}}^{2 \pi}\left(1+\cos \frac{\theta_{2}}{2}\right)\left(1-\cos \frac{\theta_{2}-\theta_{1}}{2}\right) d \theta_{2} \\
& =\frac{1}{2}\left(1-\cos \frac{\theta_{1}}{2}\right)\left[\theta_{2}+2 \sin \frac{\theta_{2}}{2}-2 \sin \frac{\theta_{2}-\theta_{1}}{2}\right]_{\theta_{1}}^{2 \pi}-\frac{1}{2}\left(1-\cos \frac{\theta_{1}}{2}\right) \int_{\theta_{1}}^{2 \pi} \frac{1}{2}\left(\cos \frac{\theta_{1}}{2}+\cos \left(\theta_{2}-\frac{\theta_{1}}{2}\right)\right) d \theta_{2} \\
& =\frac{1}{2}\left(1-\cos \frac{\theta_{1}}{2}\right)\left(2 \pi-\theta_{1}-3 \sin \frac{\theta_{1}}{2}-\pi \cos \frac{\theta_{1}}{2}+\frac{\theta_{1}}{2} \cos \frac{\theta_{1}}{2}\right) .
\end{aligned}
$$

Similarly,

$$
J_{2}=\frac{1}{2}\left(1+\cos \frac{\theta_{1}}{2}\right) \int_{0}^{\theta_{1}}\left(1-\cos \frac{\theta_{2}}{2}\right)\left(1-\cos \frac{\theta_{2}-\theta_{1}}{2}\right) d \theta_{2}=\frac{1}{2}\left(1+\cos \frac{\theta_{1}}{2}\right)\left(\theta_{1}-3 \sin \frac{\theta_{1}}{2}+\frac{\theta_{1}}{2} \cos \frac{\theta_{1}}{2}\right) .
$$

Calculation shows that

$$
\int_{0}^{\pi} J_{1} d \theta_{1}=\int_{0}^{\pi} \frac{1}{2}\left(1-\cos \frac{\theta_{1}}{2}\right)\left(2 \pi-\theta_{1}-3 \sin \frac{\theta_{1}}{2}-\pi \cos \frac{\theta_{1}}{2}+\frac{\theta_{1}}{2} \cos \frac{\theta_{1}}{2}\right) d \theta_{1}=-\frac{17}{4}-\frac{3}{2} \pi+\frac{15}{16} \pi^{2} .
$$

and

Hence

$$
\int_{0}^{\pi} J_{2} d \theta_{1}=\int_{0}^{\pi} \frac{1}{2}\left(1+\cos \frac{\theta_{1}}{2}\right)\left(\theta_{1}-3 \sin \frac{\theta_{1}}{2}+\frac{\theta_{1}}{2} \cos \frac{\theta_{1}}{2}\right) d \theta_{1}=-\frac{31}{4}+\frac{3}{2} \pi+\frac{5}{16} \pi^{2} .
$$

$$
\overline{r^{2}}=\frac{2}{\pi^{2}}\left[\int_{0}^{\pi} J_{1} d \theta_{1}+\int_{0}^{\pi} J_{2} d \theta_{1}\right]=\frac{5}{2}-\frac{24}{\pi^{2}}=0.06829 \ldots
$$

and we get the variance of the inradius.

Formula 2.

$$
\operatorname{var}(r)=\overline{r^{2}}-\bar{r}^{2}=\frac{3}{2}-\frac{144}{\pi^{4}}=0.02169 \ldots
$$

\section{Triangles of unit perimeter}

Consider those triangles, whose perimeter is equal to 1 . Our goal is to calculate the average area, average inradius and average circumradius of these triangles. The first question is, how to choose a random triangle $A B C$ of sides $a, b, c$ with $P(\triangle A B C)=a+b+c=1$ ? Note that necessary and sufficient conditions for $a, b, c$ to generate such a triangle are $a, b, c>0$, $a+b+c=1$ and triangle inequalities $a+b>c, a+c>b, b+c>a$. These necessary and sufficient conditions become

$$
a+b+c=1 \text { and } 0<a, b, c<\frac{1}{2}
$$

Our method of random choosing is the following. Choose number $a$ uniformly from $\left(0, \frac{1}{2}\right)$, then choose number $b$ uniformly from $\left(\frac{1}{2}-a, \frac{1}{2}\right)$ and then fix $c=1-a-b$. This method ensures that (2.1) holds. Then the average value of area

$$
A(\triangle A B C)=\frac{1}{4} \sqrt{(a+b+c)(-a+b+c)(a-b+c)(a+b-c)}=\frac{1}{4} \sqrt{(1-2 a)(1-2 b)(2 a+2 b-1)}
$$

given by Heron's formula is

Calculation [3] gives us

$$
\bar{A}=8 \int_{0}^{\frac{1}{2}} \frac{\sqrt{1-2 a}}{4}\left[\int_{\frac{1}{2}-a}^{\frac{1}{2}} \sqrt{(1-2 b)(2 a+2 b-1)} d b\right] d a .
$$

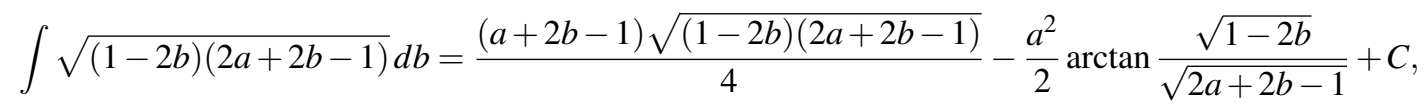

whence

$$
\int_{\frac{1}{2}-a}^{\frac{1}{2}} \sqrt{(1-2 b)(2 a+2 b-1)} d b=\frac{\pi}{4} a^{2} .
$$




\section{Formula 3.}

$$
\bar{A}=\frac{\pi}{2} \int_{0}^{\frac{1}{2}} \sqrt{1-2 a} a^{2} d a=\frac{\pi}{105}=0.02991 \ldots
$$

One can easily obtain

$$
\overline{A^{2}}=8 \int_{0}^{\frac{1}{2}} \frac{1-2 a}{16}\left[\int_{\frac{1}{2}-a}^{\frac{1}{2}}(1-2 b)(2 a+2 b-1) d b\right] d a=\frac{1}{960},
$$

and then the variance of the area.

\section{Formula 4.}

$$
\operatorname{var}(A)=\overline{A^{2}}-\bar{A}^{2}=\frac{1}{960}-\frac{\pi^{2}}{11025}=0.000146 \ldots
$$

Since the inradius of triangle $A B C$ is $r(\triangle A B C)=\frac{A(\triangle A B C)}{\frac{P(\triangle A B C)}{2}}=2 A(\triangle A B C)$, we can easily obtain the average value and the variance of the inradius.

\section{Formula 5.}

$$
\begin{aligned}
\bar{r} & =\frac{2 \pi}{105}=0.05983 \ldots \\
\operatorname{var}(r) & =\overline{r^{2}}-\bar{r}^{2}=\frac{4}{960}-\frac{4 \pi^{2}}{11025}=0.000585 \ldots
\end{aligned}
$$

The circumradius of triangle $A B C$ is equal to $R(\triangle A B C)=\frac{a b c}{4 A(\triangle A B C)}$. Therefore, the average value of the circumradius is

$$
\bar{R}=8 \int_{0}^{\frac{1}{2}} \frac{a}{\sqrt{1-2 a}}\left[\int_{\frac{1}{2}-a}^{\frac{1}{2}} \frac{b(1-a-b)}{\sqrt{(1-2 b)(2 a+2 b-1)}} d b\right] d a .
$$

After some calculation [3] we have

$$
\int \frac{b(1-a-b)}{\sqrt{(1-2 b)(2 a+2 b-1)}} d b=\frac{a^{2}-4 a+2}{16} \arctan \frac{a+2 b-1}{\sqrt{(1-2 b)(2 a+2 b-1)}}+\frac{(a+2 b-1) \sqrt{(1-2 b)(2 a+2 b-1)}}{16}+C .
$$

From this, we obtain

$$
\int_{\frac{1}{2}-a}^{\frac{1}{2}} \frac{b(1-a-b)}{\sqrt{(1-2 b)(2 a+2 b-1)}} d b=\frac{\pi}{16}\left(a^{2}-4 a+2\right) .
$$

Finally, we get the average value of the circumradius.

\section{Formula 6.}

$$
\bar{R}=\frac{\pi}{2} \int_{0}^{\frac{1}{2}} \frac{a\left(a^{2}-4 a+2\right)}{\sqrt{1-2 a}} d a=\frac{2 \pi}{21}=0.29919 \ldots
$$

Note that since

$$
\overline{R^{2}}=8 \int_{0}^{\frac{1}{2}} \frac{a^{2}}{1-2 a}\left[\int_{\frac{1}{2}-a}^{\frac{1}{2}} \frac{b^{2}(1-a-b)^{2}}{(1-2 b)(2 a+2 b-1)} d b\right] d a
$$

does not converge, the circumradius has infinite variance.

\section{Conclusion}

Our main aim in this study was to examine the average area, perimeter, inradius, circumradius of triangles. One approach for considering certain triangles is to fix their circumradiuses to 1 . Then the average area and perimeter of triangles were already known to be $\frac{3}{2 \pi}$ and $\frac{12}{\pi}$. In the paper, we calculated the average inradius and the variance being $\frac{12}{\pi^{2}}-1$ and $\frac{3}{2}-\frac{144}{\pi^{4}}$. Another approach is to consider triangles of unit perimeter. We calculated the average area, inradius and circumradius of such triangles being $\frac{\pi}{105}, \frac{2 \pi}{105}$ and $\frac{2 \pi}{21}$ along with the accompanying variances. A possible extension of these results may be the calculation of the average values of polygons with more than three sides.

\section{References}

[1] A. Madras, S. KC, Randomly generated triangles whose vertices are vertices of regular polygons, Rose-Hulman Undergrad. Math. J., 7(2) (2006),

[2] E. W. Weistein, Circle triangle picking. From MathWorld - A Wolfram Web Resource. http://mathworld.wolfram.com/ CircleTrianglePicking.html

[3] Wolfram Alpha LLC, 2018, Wolfram|Alpha. https://www.wolframalpha.com 